\title{
Data Pattern Dependence of VCSEL Far-Field Distributions
}

\author{
S. Al-Sowayan and K. L. Lear, Member, IEEE
}

\begin{abstract}
The far field divergence angle distribution of verticalcavity surface-emitting lasers is found to exhibit dependence on the data pattern driving the lasers. Two $50 \%$ duty cycle $1.25-\mathrm{Gb} / \mathrm{s}$ data patterns chosen to cause the same thermal conditions but with frequency content differing by a factor of 16 resulted in changes in the beam profile distributions with up to a $30 \%$ power variation in the central on-axis portion of the beam. Examination of the temporal waveforms as a function of far field angle revealed overshoot in the on-axis power and undershoot in the off-axis portion of the beam.
\end{abstract}

Index Terms-Beam divergence, data pattern dependent, far field, laser diode, multimode fiber optic.

\section{INTRODUCTION}

$\mathbf{V}$ ERTICAL-CAVITY surface-emitting lasers (VCSELs) and multimode fibers (MMFs) are widely used in local and storage area networks. As network data rates increase, bandwidth limitations of MMF can severely limit link distances [1]. Practical MMF with 62.5- $\mu \mathrm{m}$ core diameter and 850-nm wavelength can have bandwidth as low as $160 \mathrm{MHz} \cdot \mathrm{km}$, and the observed bandwidth can be sensitive to launch conditions. An overfilled launch (OFL) condition such as that produced by light-emitting diodes results in a low but stable bandwidth condition that is insensitive to source to fiber misalignment. However, laser-based transmitters do not achieve the OFL condition [2], [3]. In particular, on-axis power excites a selection of low-order modes which results in more pulse broadening relative to the OFL condition [3] due to a common defect of having a distinct peak or dip in the index of refraction profile at the center of the fiber as illustrated in the inset of Fig. 1. The body of Fig. 1 shows the dependence of bandwidth on the launch angle for two different 850-nm source-to-fiber offsets based on data from a theoretical study of MMF dispersion [4]. For example, if there is a tilt of $2^{\circ}-3.5^{\circ}$ or an offset of $3 \mu \mathrm{m}$, the bandwidth would be less than the OFL bandwidth, which is $366 \mathrm{MHz} \cdot \mathrm{km}$ in this case.

Since the bandwidth is sensitive to launch angle, it is important to consider which group of modes are being excited by laser sources. This letter first reports beam profile measurements showing that VCSEL beam divergence angle distributions are data pattern dependent even if these data patterns have the same thermal content. Next, the average beam profile is related to the temporal shape of the output pulses as a function of the divergence angle. The letter concludes with consideration of implications of pattern-dependent beam profiles of VCSEL sources on dispersion in launch angle-dependent MMF.

Manuscript received November 12, 2003; revised June 2, 2004.

The authors are with the Department of Electrical and Computer Engineering, Colorado State University, Fort Collins, CO 80523 USA (e-mail: sowayan@engr.colostate.edu; kllear@engr.colostate.edu).

Digital Object Identifier 10.1109/LPT.2004.833925

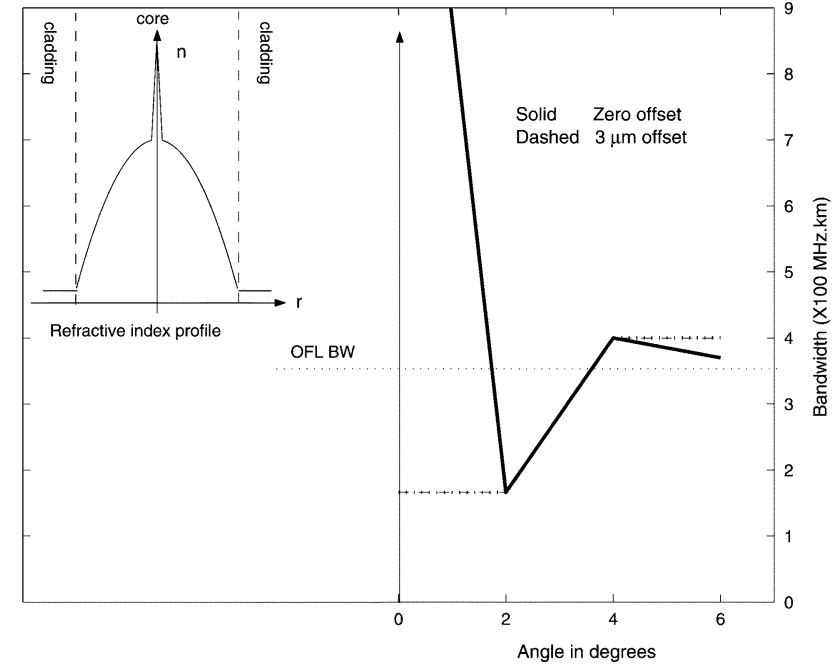

Fig. 1. Refractive index profile for MMF fibers with a central peak (inset), bandwidth dependence on angle of launch and laser to fiber offset (reproduced after [4] for 850-nm transmission.).

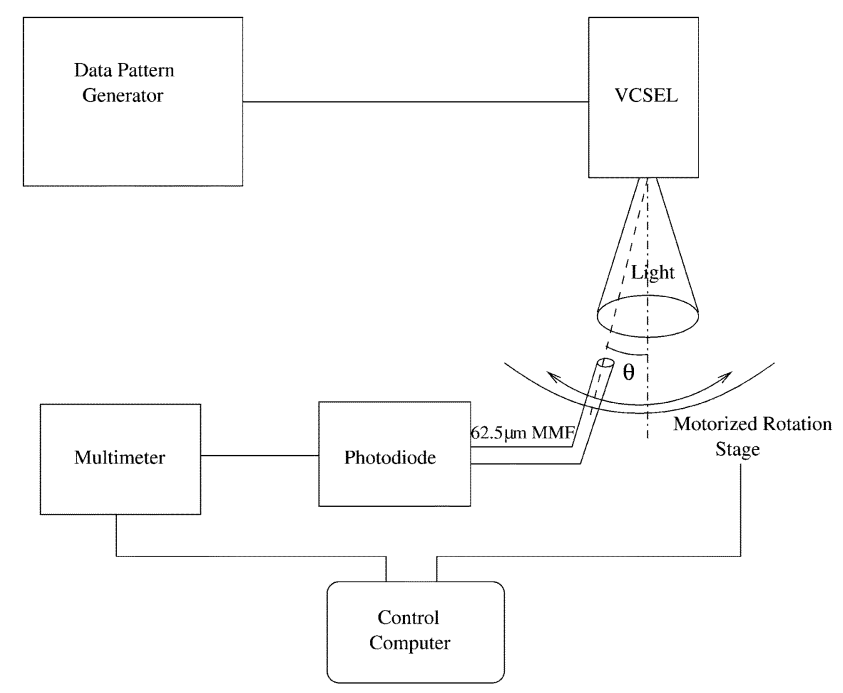

Fig. 2. Experimental setup.

\section{Beam Profile Measurements}

Commercial VCSELs purchased from two major VCSEL manufacturers have been used as the optical sources in the experimental setup of Fig. 2. Parts from both manufacturers, referred to as Part A and Part B, are oxide-confined 2.5-Gb/s 850-nm VCSELs in transmitter optical subassembly (TOSA) packages with an approximately $1: 1$ imaging lens. Multiple samples of each part were tested with consistent results. The VCSELs are directly driven by a bit-error-ratio tester (Agilent 


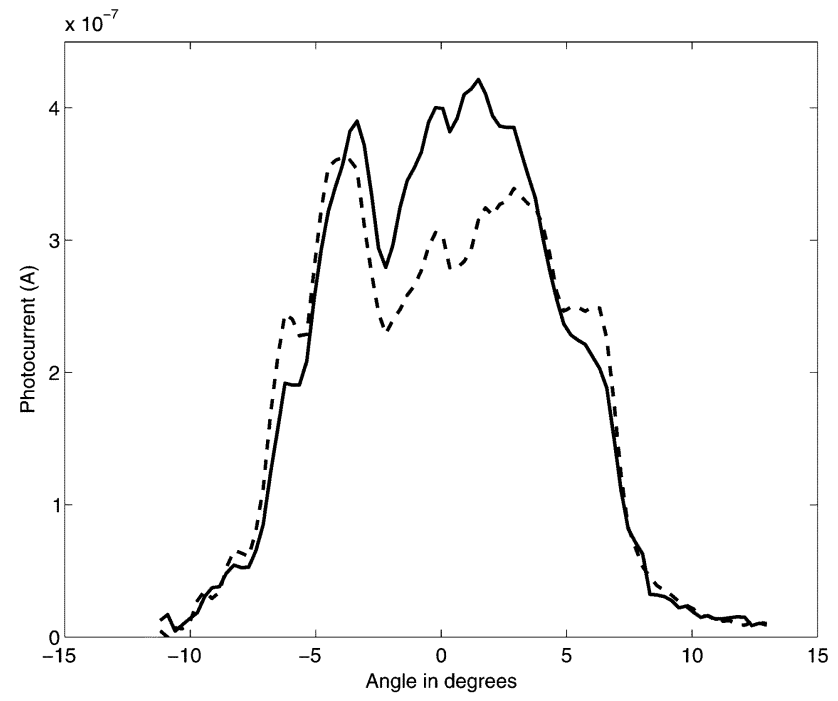

Fig. 3. Beam divergence angle distribution for Part A: alternating ones and zeros pattern (solid), alternating 16 ones and 16 zeros (dashed).

81250 ) acting as a $50-\Omega$ source at a rate of $1.25 \mathrm{~Gb} / \mathrm{s}$. The high and low drive levels were set to achieve an extinction ratio of approximately $13 \mathrm{~dB}$ and average total power of $-4 \mathrm{dBm}$, which are typical values for gigbits/s MMF links. The output beam divergence angle distribution is measured by rotating a cleaved MMF tip around the focal spot of the VCSEL TOSA under test. The fiber is connected to a photodiode to measure the received power. The distance between the fiber tip and the TOSA focal spot is approximately $5 \mathrm{~mm}$ so that the $62.5-\mu \mathrm{m}$ core diameter of the fiber subtends an angle of $0.7^{\circ}$. The rotation stage is motorized and is controlled by a computer that also obtains the photocurrent as the fiber tip is stepped through the beam angles. A rotation stage was used instead of a translation stage to maintain constant light acceptance of the fiber independent of position. The beam divergence angles from the TOSA focal spot are assumed to be comparable to those from the VCSEL itself due to the $1: 1$ imaging ratio of the lens in the TOSA. The VCSELs were driven with two different square wave data patterns, one with a 2-bit period and one with a 32-bit period corresponding to fundamental frequencies of 625 and $39.1 \mathrm{MHz}$, respectively. Both patterns had a 50\% duty cycle so that the average power dissipation and, thus, thermal biasing were the same. The maximum run length of 16 ones or zeroes for the low frequency pattern exceeds the run length for Gigabit Ethernet's 8 B/10 B block coding, but higher data rate standards, e.g., 10-Gb Ethernet, may use coding with run lengths of up to 64 [5].

The measured far field distribution of Part A is shown in Fig. 3, where the solid line is for the high frequency pattern, and the dashed line is for the low frequency pattern. The far field scan was repeated four times and found to be reproducible with Fig. 3 being the average of the four. It is clear that the high frequency pattern results in 30\% higher on-axis output power and $25 \%$ lower off-axis output power in comparison to the low frequency pattern. The situation is similar for Part B shown in Fig. 4, where the on-axis power is slightly higher for the high frequency patterns but the difference is not as great as for Part $\mathrm{A}$, and both high and low frequency patterns have the notable side lobes at $\pm 7.5^{\circ}$. Patterns with different duty cycles could

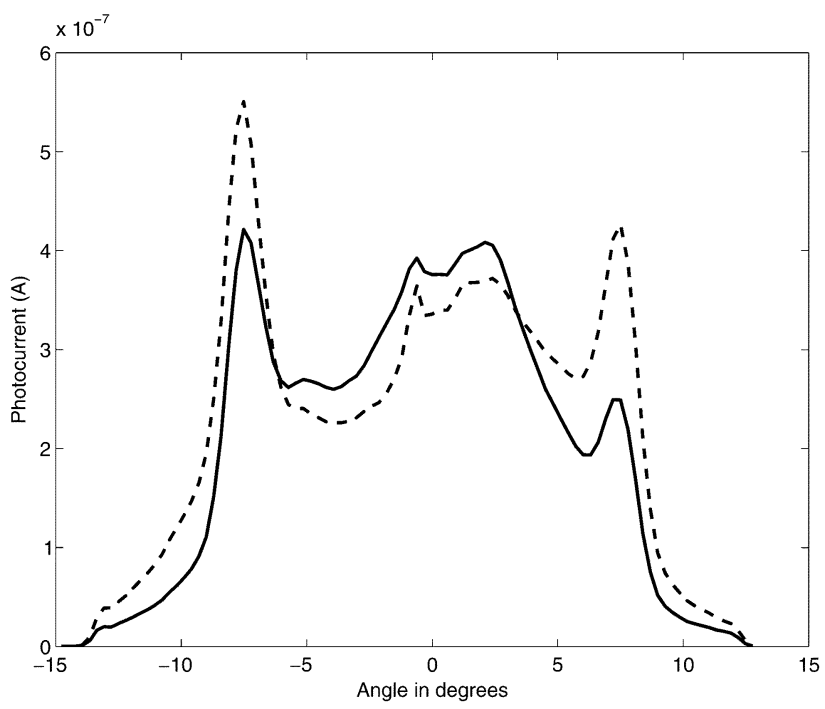

Fig. 4. Beam divergence angle distribution for Part B: alternating ones and zeros pattern (solid), alternating 16 ones and 16 zeros (dashed).
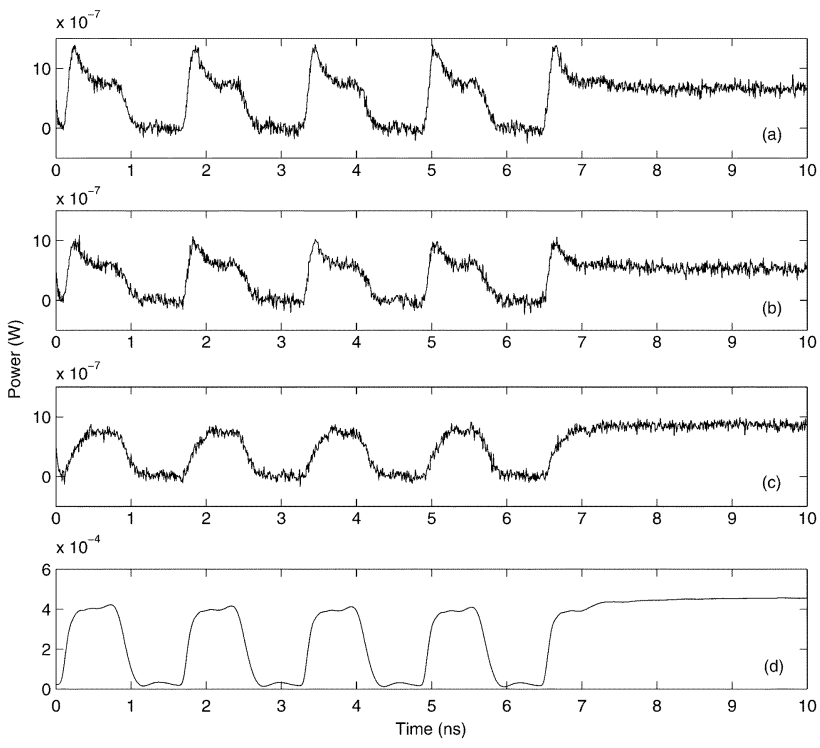

Fig. 5. Bit time pulses of Part A: (a) at $0^{\circ}$; (b) at $-3^{\circ}$; (c) at $-6^{\circ}$; and (d) total integrated power.

be expected to change the power dissipation and, thus, strength of the thermal lens which in turn would impact the beam divergence slightly for oxide-confined VCSELs and to a greater extent in implant-confined VCSELs [6]. But the effects observed here with the same duty cycles must be related to carrier dynamics on a time scale much shorter than the thermal time constant.

\section{TEMPORAL WAVEFORM MEASUREMENTS}

To better understand the origin of the change in beam distribution with data pattern, the temporal waveform of the laser output was observed as a function of far field angle using a data pattern with both high and low frequency content. This measurement was done by coupling the fiber patch cord to a Hewlett Packard model 86 100A digital oscilloscope with a face contact optical channel plugin model 86101A. The bandwidth of the optical channel was specified to be at least $3 \mathrm{GHz}$. Fig. 5 shows the 
measured pulse shapes for Part A at launch angles of [Fig. 5(a)] at $0^{\circ}$, [Fig. 5(b) $]-3^{\circ}$, and [Fig. 5(c)] $-6^{\circ}$.

These waveforms show up to $85 \%$ overshoot on-axis and undershoot off-axis at the zero to one transition. Part B produces very similar waveforms, but with $60 \%$ overshoot. For both parts, overshoot is maximized at $0^{\circ}$ (directly on-axis), and the overshoot contributes noticeably to the average on-axis power from the VCSEL. The presence of more transitions in the high frequency pattern results in it generating more power on-axis than the low frequency pattern. Part B's slightly reduced overshoot in comparison to Part A corresponds to a smaller variation in average on-axis power. When the fiber is placed into the TOSA to couple all of the light within the numerical aperture of the fiber, both Parts A and B create waveforms, as shown in Fig. 5(d), with significantly less overshoot or undershoot than seen in the angle resolved waveforms. Thus, the on-axis overshoot and off-axis undershoot in large part balance when the integrated power is considered.

\section{DisCUSSION OF RESUlTS}

Prior investigations of polarization resolved waveforms led the authors of [7] to conclude that a low-to-high drive transition on an implant-confined VCSEL caused the high-order mode to overshoot and the low-order mode to undershoot. With the assumption that the fundamental mode contributes more on-axis power and higher order modes contribute more power in the wings of the beam distribution, it appears that the oxideconfined devices in this study behave opposite to those in [7]. Here, the fundamental mode appears to overshoot and highorder mode undershoots. The difference in the angle and polarization resolved studies might be due to the different behaviors of implant and oxide confined VCSELs. The strong coupling of the high-frequency edge transition to the fundamental mode observed in this study is not consistent with a peripheral current crowding effect as seen in bipolar transistor emitters, and the duration of the overshoot is inconsistent with thermal timescales. One possible cause for the observed overshoot is spatial hole burning decreasing the central carrier density and thus decreasing fundamental mode power as the photon density builds after the zero to one transition.

Since the angularly integrated waveforms of Fig. 5(d) show minimal data pattern dependence, the VCSEL sources investigated here would not be expected to significantly contribute to data pattern dependence of an MMF system if the optical power launched at all angles propagated with comparable delay and dispersion. However, the studies referenced in the introduction indicate that MMF commonly exhibits angle-dependent dispersion. If a source with data pattern-dependent launch angle is coupled to a fiber with launch angle-dependent dispersion, there exists the likelihood that the fiber link will exhibit data pattern-dependent dispersion unless the chained dependencies fortuitously and improbably cancel.

The angle resolved waveforms with undershoot or overshoot can be considered a nonlinear response to the fairly rectangular current pulses. To the extent that the fiber bandwidth or corresponding impulse response varies with launch angle, as shown in Fig. 1, the overall source and fiber system could behave nonlinearly. This situation is aggravated by the strong on-axis over- shoot coupling to the least stable bandwidth region at the center of the fiber. The increasing speed of VCSEL sources combined with the limited bandwidth of conventional MMF has generated interest in equalization of dispersion in MMF in order to obtain useful link distances [8]. The experimental results presented here indicate that optimal equalization of VCSEL-based MMF systems may require their treatment as nonlinear and data pattern dependent.

The encircled flux criterion has been suggested to avoid launch conditions that could aggravate anomalous MMF bandwidth and might reduce the consequences of the data pattern dependency. However, some researchers feel it will not prevent the effect of the refractive index defects in fibers [9]. Further, the present criterion addresses offset rather than angle, and encircled flux is typically measured without modulation, so it is not clear that adherence to the encircled flux criterion would prevent the phenomena discussed above.

\section{SUMMARY}

Experiments described here demonstrate that VCSEL beam profile distributions are data pattern dependent, even if the patterns result in the same thermal conditions. Near the on-axis angles, the average power is higher in high-frequency patterns because of the on-axis overshoot in the zero-to-one transitions. High-frequency patterns result in undershoot and, thus, lower power at larger beam angles. The resulting nonlinear data-dependent response of MMF systems with launch angle-dependent bandwidth may impact equalization processes for such systems. Future studies are planned to quantify the effect of this phenomenon on data link performance.

\section{REFERENCES}

[1] L. Raddatz and I. H. White, "Overcoming the modal bandwidth limitation of multimode fiber by using passband modulation," IEEE Photon. Technol. Lett., vol. 11, pp. 266-268, Feb. 1999.

[2] L. Raddatz, I. H. White, D. G. Gunningham, and M. C. Nowell, "Influence of restricted mode excitation on bandwidth of multimode fiber links," IEEE Photon. Technol. Lett., vol. 10, pp. 534-536, Apr. 1998.

[3] L. J. Sargent, M. Webster, I. H. White, P. J. Heard, R. V. Penty, M. R. T. Tan, and D. G. Cunningham, "Simple technique for bandwidth enhancement of multimode fiber links using controlled spatial emission from vertical cavity surface emitting lasers," Electron. Lett., vol. 34, pp. 2038-2040, Oct. 1998.

[4] M. Webster, L. Raddatz, I. H. White, and D. G. Cunningham, "A statistical analysis of conditioned launch for gigabit ethernet links using multimode fiber," J. Lightwave Technol., vol. 17, pp. 1532-1541, Sept. 1999.

[5] R. Walker and R. Dugan. Low overhead coding proposal $10 \mathrm{GbE}$ serial links. presented at IEEE802.3 Higher Speeed Study Group Plenary Week Meeting. [Online]. Available: http://group.ieee.org/groups/802/3/10G_study/public/nov99/index.html

[6] K. L. Lear, R. P. Schneider, Jr., K. D. Choquette, and S. P. Kilcoyne, "Index guiding dependent effects in implant and oxide confined verticalcavity lasers," IEEE Photon. Technol. Lett., vol. 8, pp. 740-742, June 1996.

[7] D. V. Kuksenkov, H. Temkin, and S. Swirhun, "Polarization instability and relative intensity noise in vertical-cavity surface-emitting lasers," Appl. Phys. Lett., vol. 67, pp. 2141-2143, Oct. 1996.

[8] K. M. Patel and S. E. Ralph, "Enhanced multimode fiber link performance using a spatially resolved receiver," IEEE Photon. Technol. Lett., vol. 14, pp. 393-395, Mar. 2002.

[9] C. A. Bung, J. R. Kropp, and K. Petermann, "Reliability of power distribution condition for 10-Gigabit-Ethernet transmission," in Proc. LEOS, 2001, pp. 290-291. 